\title{
Weaning of rotary blood pump recipients after myocardial recovery: A computer study of changes in cardiac energetics
}

\author{
Heinrich Schima, $\mathrm{PhD}^{\mathrm{a}, \mathrm{b}}$ \\ Michael Vollkron, $\mathrm{MS}^{\mathrm{a}, \mathrm{c}}$ \\ Herbert Boehm, MS ${ }^{b}$ \\ Wilfried Röthy, MD \\ Markus Haisjackl, MD ${ }^{d}$ \\ Georg Wieselthaler, MD \\ Ernst Wolner, MD ${ }^{a}$
}

\footnotetext{
From the Departments of Cardio-Thoracic Surgery and LBI of Cardio-Surgical Research, ${ }^{a}$ Biomedical Engineering and Physics, ${ }^{\mathrm{b}}$ Biomedical Research, ${ }^{\mathrm{c}}$ Cardiovascular Anesthesiology, ${ }^{\mathrm{d}}$ University of Vienna, Austria.

Received for publication May 26, 2003; revisions requested July 7, 2003; accepted for publication Sept 9, 2003.

Address for reprints: Heinrich Schima, $\mathrm{PhD}$, Institute of Biomedical Research, University of Vienna, Waehringer Guertel 18-1Q, A-1090 Vienna, Austria (E-mail: heinrich.schima@akh-wien.ac.at).

J Thorac Cardiovasc Surg 2004;127: $1743-50$

$0022-5223 / \$ 30.00$
}

Copyright () 2004 by The American Association for Thoracic Surgery

doi:10.1016/j.jtcvs.2003.09.029
Background: Weaning of patients from mechanical cardiac support after myocardial recovery has always involved multiple, interacting factors, particularly the training of the myocardium during reduction of pump flow. Rotary pumps offer training advantages when support flow is reduced, even to nearly zero. We report a computer analysis that evaluates the work required of the heart during partial unloading and removal of rotary pumps.

Methods and Results: A computer model of the assisted circulation, previously implemented in MATLAB (The MathWorks Inc, Natick, Mass), has been augmented with a model of the MicroMed DeBakey ventricular assist device (MicroMed Technology, Inc, Houston, Tex). Flow, pressure patterns, and external work (pressure-volume area, calculated as the area of the ventricular pressure-volume loop [external work] plus potential energy) were calculated for nonassisted and various continuously assisted patients. Under low-flow conditions, the heart imposes an oscillating forward-backward flow through the non-occlusive rotary pump, causing an increase in ventricular work. Thus, an assist flow of 1 to $1.5 \mathrm{~L} / \mathrm{min}$ requires work equivalent to that of the unsupported heart. At $60 \%$ contractility, the nonassisted pressure-volume area is $1.10 \mathrm{Ws} / \mathrm{beat}$, and the potential energy is 0.38 $\mathrm{Ws} /$ beat. At a Qpump of $1 \mathrm{~L} / \mathrm{min}$, the pressure-volume area is $1.21 \mathrm{Ws} /$ beat, and the potential energy is $0.37 \mathrm{Ws} /$ beat. At a Qpump of $3 \mathrm{~L} / \mathrm{min}$, the pressure-volume area is $0.93 \mathrm{Ws} /$ beat, and the potential energy is $0.29 \mathrm{Ws} /$ beat. These conditions cannot be achieved with pulsatile systems.

Conclusion: During weaning and retraining, an implanted rotary pump can provide a workload to the heart like that in the nonassisted situation, thus increasing the predictability of weaning and reducing the risk of reiterating heart failure.

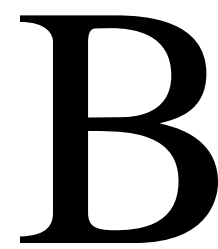

ridging the failing heart to myocardial recovery has always been a key indication for the application of mechanical cardiac assist devices and has become more relevant with the shortage of donor grafts for transplantation in recent years. Various reasons for cardiac failure, including acute inflammation, autoimmune disease, and long-term cellular degradation suggest different therapeutic approaches, ${ }^{1-4}$ but the number of successful withdrawals is still low. New options such as stem cell therapy and the use of neoangiogenic agents may increase the number of successful withdrawals. ${ }^{5-7}$

However, the determination of when to withdraw assistance and explant the assist device has always been crucial. From the standpoint of myocardial retraining 
and reliable evaluation of myocardial capacity, driving the pump at low-or even zero-flow would be highly desirable. However, in pulsatile systems, a flow less than approximately $3 \mathrm{~L} / \mathrm{min}$ is correlated with a greatly increased risk of thrombus formation, and a flow near zero is absolutely prohibited for times exceeding several minutes. Sophisticated protocols and diagnostic parameters have therefore been developed to assess recovered heart function during short interruptions of assist to evaluate the possibility of weaning. ${ }^{1,3,8}$ With the paracorporeal rotary blood pumps now widely used, along with their long tubes, a flow less than $1.5 \mathrm{~L} / \mathrm{min}$ for extended time periods is likely to raise the risk of embolization because of extended particle residence time and reduced washout.

In contrast with these devices, implanted axial pumps, because of their design and shorter cannulas, have less blood-contacting surface and fewer stagnation points. Because of lower hydraulic inertia and friction loss in these short cannulas, blood flow acquires a greater pulsatile component after initial cardiac recovery. ${ }^{9,10}$ In the case of pump stoppage, the natural heart generates a leakage flow through the pump that is equivalent to a medium-grade aortic insufficiency. One group even suggests that it is possible to stop the device for extended time periods, ${ }^{11,12}$ although the potential thrombogenicity of a stationary mechanical bearing has not been examined in detail.

The features of axial pumps allow reducing pump flow for extended times and therefore make them excellent candidates for patients who appear capable of ventricular recovery and weaning. As mentioned previously, the number of such patients may substantially increase when new therapies aiding recovery after massive myocardial infarction become available.

However, if such pumps are adjusted to low rotational speeds and mean flows near zero, forward-backward flow pulsations through the pump will cause considerable positive and negative instantaneous values of flow. Consistent with these observations, some currently available controllers do not allow speed to be adjusted below a set level for reasons of safety. These flow conditions are already somewhat equivalent to aortic valve insufficiency, because they require the heart to eject an additional volume during systole that is returned by backflow through the pump during diastole. Thus, a mean flow of zero is not equivalent in an unassisted patient as it would be in a patient with a pulsatile, valved assist device, in which pump stoppage causes valve closure.

Thus we hypothesized, for a patient assisted with a rotary pump, that a flow considerably greater than zero would already approximate the nonassisted case with respect to cardiac work and energetics. This hypothesis was addressed within our previously established and described computer model. This model also permits calculation of pressure- volume (PV) loops and the energetic parameters: external work (EW), potential energy (PE), and cumulative PV area (PVA). These, then, represent the metabolic demand of pumping $^{13-15}$ (Figure 1).

In this study, the unloading and loading effects of implanted axial pumps were calculated under weaning conditions and compared with both paracorporeal rotary blood pumps and pulsatile assist devices.

\section{Methods}

A computer model of the cardiovascular system was developed and parameterized with data obtained from the literature, and in vitro, in vivo, and clinical data were obtained by the authors as described in detail elsewhere. ${ }^{13}$ Automatic parameter variations with feedback loops (keeping selected parameters such as arterial pressure constant) and under varying states of contractility were performed.

Necessary data describing pump and cannula performance were obtained in a hydrodynamic mock loop of Donovan-Design, ${ }^{16}$ with pressure (Hellige, Freiburg, Germany) and flow (Transonic Inc, Ithaca, NY) monitoring.

Three sets of pump-cannula equations, corresponding to 3 alternative systems, were used in the current study (Figure 2). (1) Implanted rotary pump: These equations represented the hydromechanical situation imposed by a fully implanted DeBakey ventricular assist device (MicroMed Technology, Inc, Houston, Tex). The pump was positioned with a $15-\mathrm{cm}$ apical inflow cannula from the left ventricle and a 30-cm outflow cannula to the ascending aorta. (2) Paracorporeally positioned rotary pump: These equations represented the hydrodynamic effect of a paracorporeally placed pump with two $\frac{3}{8}$-inch tubes, each $1 \mathrm{~m}$ in length. (3) Pulsatile implanted system: These equations represented the hydrodynamic effect of a Novacor-Cardiac Assist Device (World Heart Inc, Ottawa, Canada) implanted abdominally with a 9-cm proprietary inflow cannula and a 30-cm outflow graft.

For this study, the model structure was simplified to focus on the interaction between the left ventricle and the pump (Figure 2). The following parameters were selected to mimic the typical condition of a clinical candidate for weaning. The peripheral resistance was adjusted to provide a constant aortic pressure of 85 $\mathrm{mm} \mathrm{Hg}$ at a systemic flow (pump flow plus cardiac flow) of 5 $\mathrm{L} / \mathrm{min}$, independent of the central venous pressure. The systemic flow of $5 \mathrm{~L} / \mathrm{min}$ was achieved by variation of venous return through pulmonary venous pressure, mimicking the clinically motivated goal of keeping the cardiac index within normal ranges. The contractility of the left ventricle was assumed to be $50 \%, 60 \%$, $70 \%, 80 \%$, or $100 \%$ of normal contractility, equivalent to ejection fractions of $34 \%, 44 \%, 50 \%, 54 \%$, and $58 \%$. These values correspond to typical ejection fractions of candidates for weaning. The heart rate was kept constant at 80 beats/min.

The rotary pumps were driven at constant speed to achieve the desired levels of assist flow (0-5 L/min, corresponding to $0 \%$ $100 \%$, in 6 steps). Pulsatile pumps were not in detail part of this study, because of their large variability in frequency, systolic time, phase, driving force, and synchronization. Therefore, for comparison, only a typical pulsatile setting for minimal allowable support 


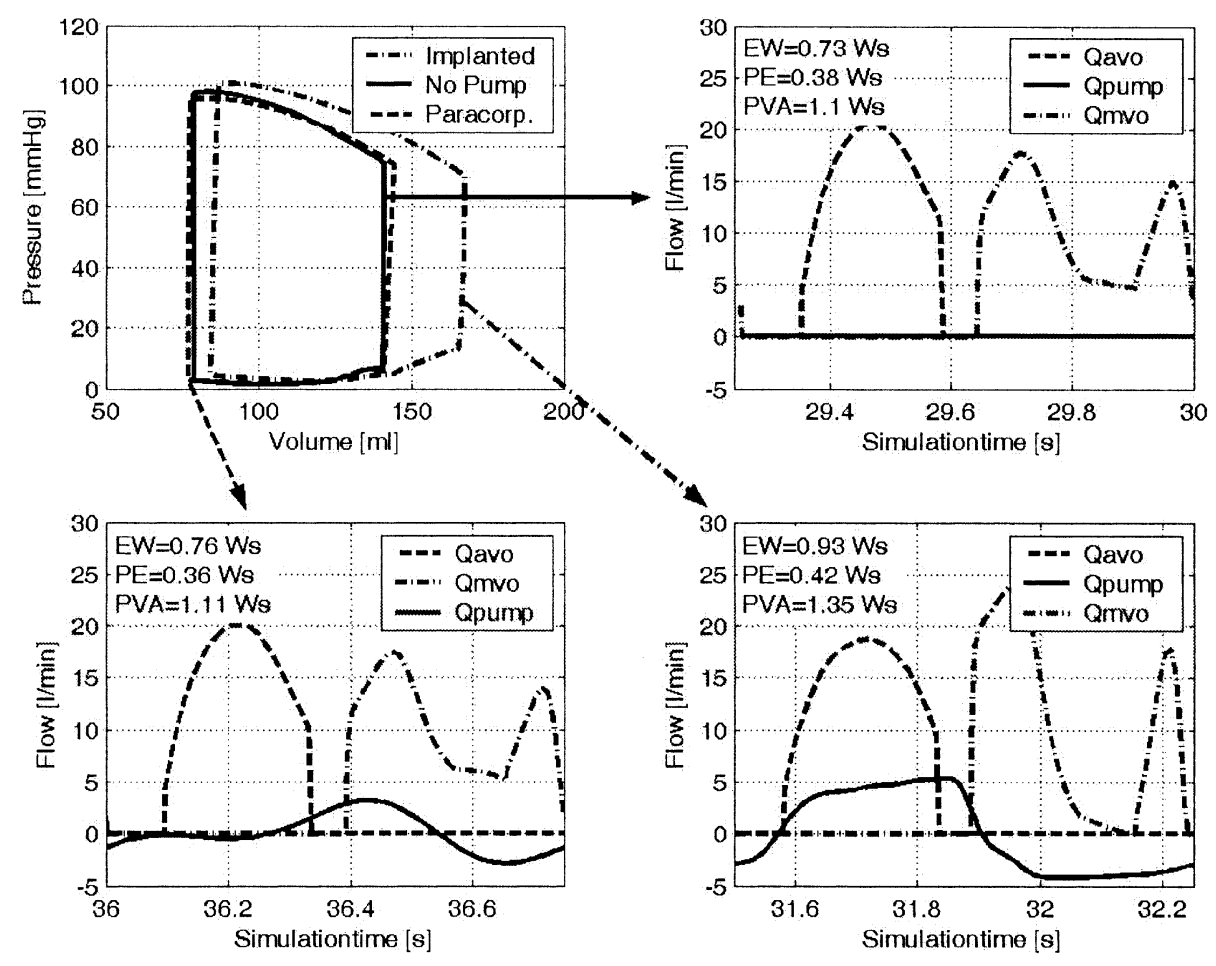

Figure 1. Comparison of flow patterns and pressure-volume (PV) loops of the unassisted left ventricle with left ventricular assistance by implanted and paracorporeally placed rotary pumps at a mean flow of $0 \mathrm{~L} / \mathrm{min}$. Compared with the unassisted heart, the flow pattern and PV loops for an implanted pump with short cannulas considerably increases the area of the PV loop because of pendulular flow. The paracorporeally positioned pump with long cannulas provides a load to the heart similar to that in the unassisted case. $E W$, External work; $P E$, potential energy; PVA, pressure-volume area; $\boldsymbol{Q}_{\text {avo }}$ flow through the aortic valve orifice; $\boldsymbol{Q}_{\text {pump }}$, flow through the pump; $\boldsymbol{Q}_{m v o^{\prime}}$ flow through the mitral valve orifice.

at normal anticoagulation was included in this study, with a $1: 2$ support mode at a pump rate of 40 beats/min.

The following parameters were calculated: ${ }^{17} \mathrm{EW}$ of the left ventricle, which corresponds to the area within the PV loop; PE, which is defined as the area within the trace of end-systolic PV relation; end-diastolic PV relation of the PV loop; and cumulative $\mathrm{PVA}=\mathrm{PE}+\mathrm{EW}$, which is considered to correspond to the whole consumption of metabolic energy per heartbeat.

\section{Results}

Figures 1, 3, and 4 show examples of flow and pressure patterns of an assisted patient with an implanted axial pump working at 3 levels $(0,1.8$, and $4.6 \mathrm{~L} / \mathrm{min})$. The waviness in pump flow caused by the cardiac contractility, the mitral flow pattern influenced by atrial contractility, and the reduced pulsatility in ventricular and aortic pressure, compared with healthy subjects, can all be seen. The slight inclinations of the PV loop during so-called isovolumetric contraction and relaxation show that these phases of the cardiac cycle are not in fact isovolumetric because of the pump flow.

Figure 5 gives the EW, PE, and resulting PVA without pump; the stopped pump with backflow; and various assist levels with a mean pump flow between 0 and $5 \mathrm{~L} / \mathrm{min}$. As hypothesized, a mean level of pump flow of zero imposes a greater load on the heart than the occluded condition. In fact, the occluded condition is equivalent, under these conditions, to a support level of approximately 1.5 to $1.8 \mathrm{~L} / \mathrm{min}$ of forward flow.

Figure 6 shows the effect of a paracorporeally placed pump and its 2 meters of tubing and cannula. Because of inertia and friction, the flow waviness is far lower than for the implanted system. Therefore there is practically no pendular volume load on the ventricle, and the loading conditions compared with the case without pump are very similar to a mean flow of zero (Figures 1 and 6). Of course, in this situation an unoccluded, stopped pump would increase cardiac load because of backflow.

Further, we tested whether changes in contractility affected the level of support flow at which the load on the heart was equal to that with no support. Within a contractility range of $60 \%$ to $80 \%$, which corresponds to an ejection fraction between $44 \%$ and $54 \%$ in the unassisted heart, no major shift of this clamp-equivalent flow was observed. This observation shows that 1.5 to $1.8 \mathrm{~L} / \mathrm{min}$ of forward 


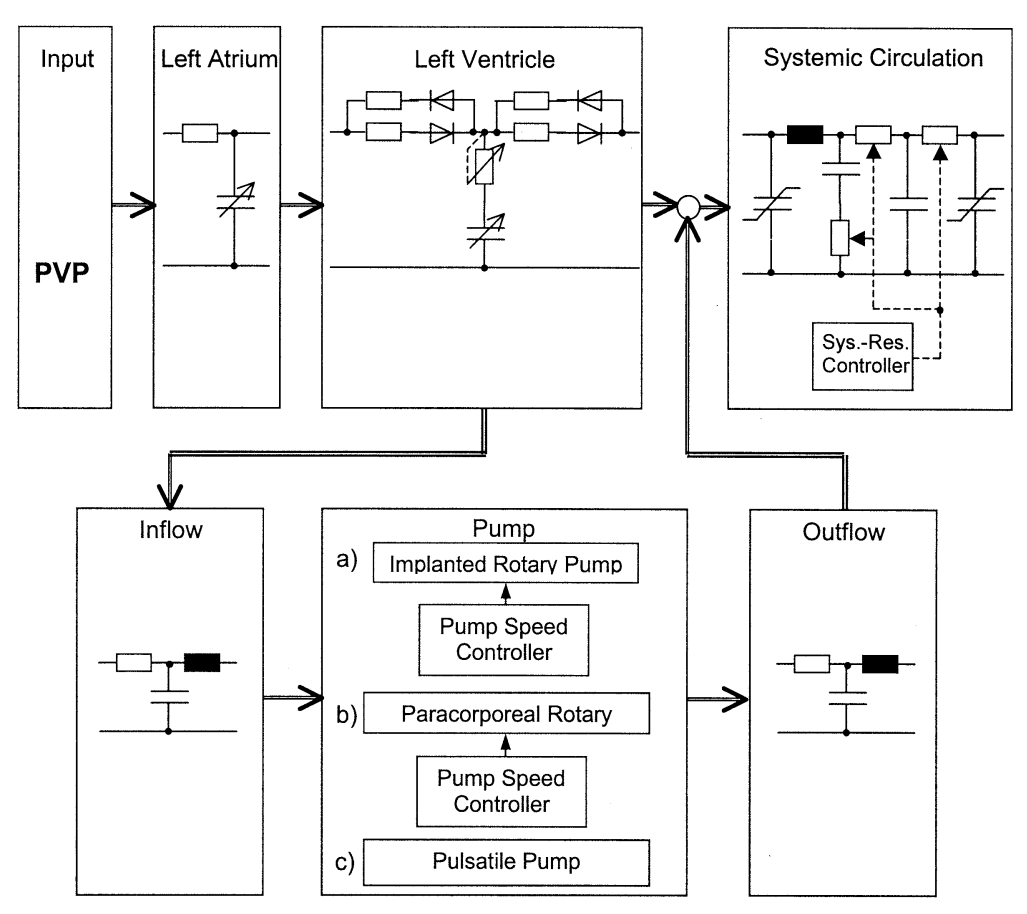

Figure 2. Model structure used for simulation.
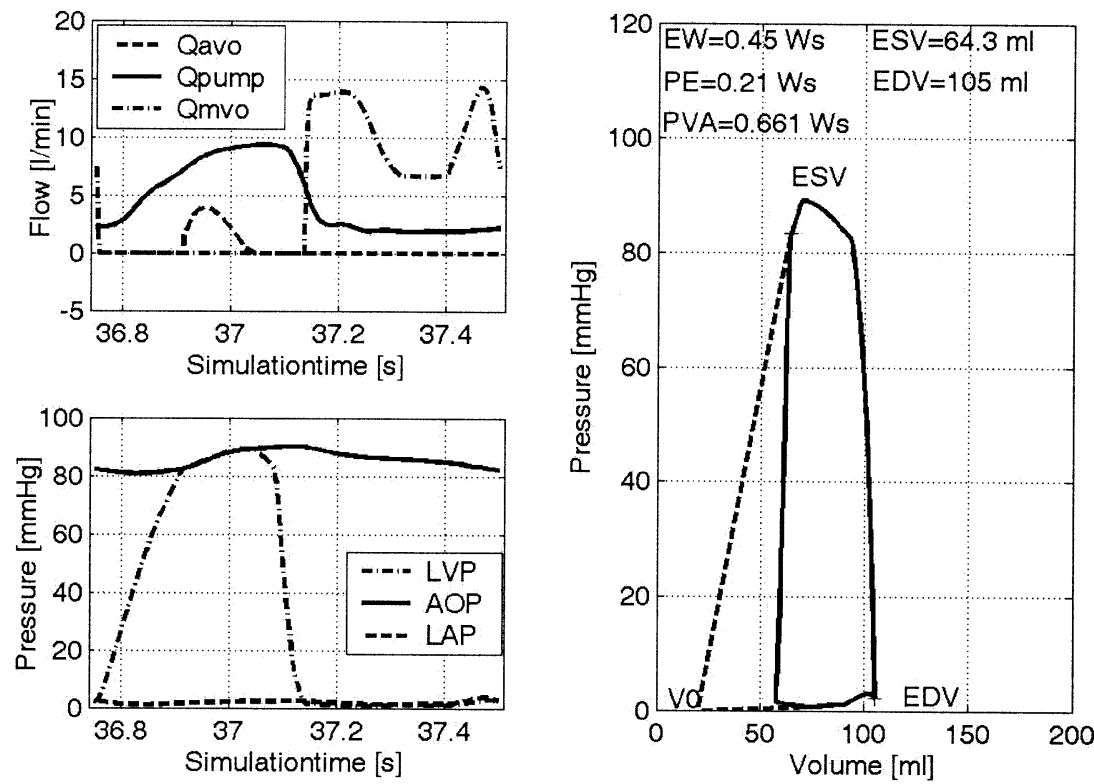

Figure 3. Pressure and flow patterns and PV loop for full assist by the implanted pump (heart rate 80 beats/min, contractility $60 \%$, pump flow $4.6 \mathrm{~L} / \mathrm{min})$. ESV, End-systolic volume; EDV, end-diastolic volume; $L V P$, left ventricular pressure; $A O P$, aortic pressure; $L A P$, left atrial pressure.

flow at an overall cardiac output of $5 \mathrm{~L} / \mathrm{min}$ (ie, $\sim 30 \%$ of total flow from the pump) would give the heart a load equivalent to an occluded system.

Even at a contractility of 50\% (33\% ejection fraction without assistance), the ventricle shows a strongly reduced capability to unload the atrium, leading to atrial pressures of more than $15 \mathrm{~mm} \mathrm{Hg}$.

In Figure 7, the condition for a pulsatile assist system is shown for a 1:1 unloading mode (ie, every second beat is assisted) at weaning. The complexity of such an unloading 

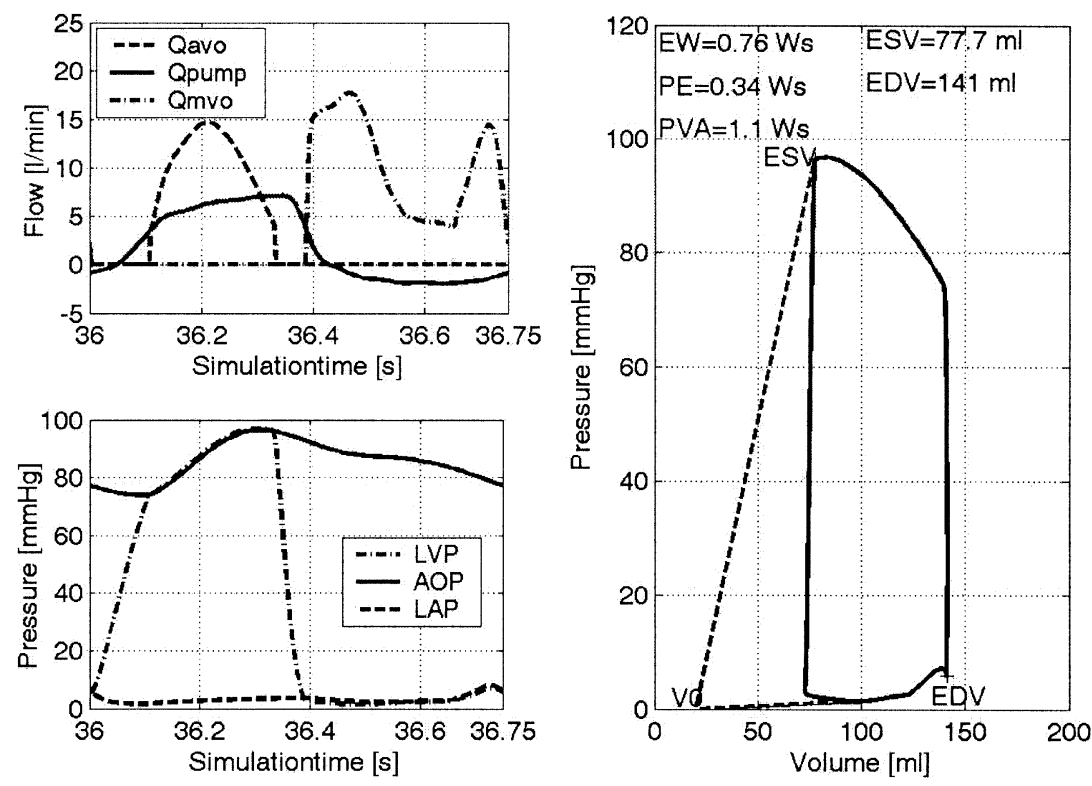

Figure 4. Pressure and flow patterns and PV loop for a mean flow of $1.8 \mathrm{~L} / \mathrm{min}$ through the implanted pump (point of load equivalent to occluded pump). The pump flow pattern $\boldsymbol{0}_{\text {pump }}$ includes a considerable backflow during diastole.

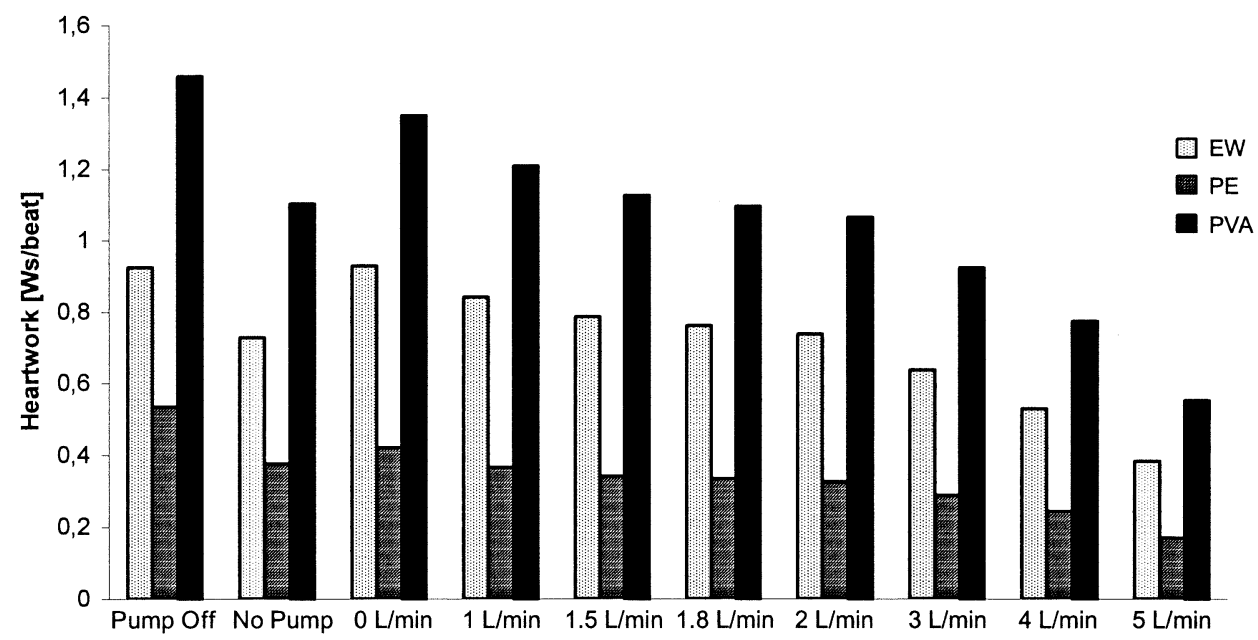

Figure 5. EW, PE, and PVA for implanted rotary pump at occlusion, zero speed, and various levels of pump flow from $0 \%$ to $100 \%$ (ie, 0-5 L/min) The PVA at pump support decreases with increasing pump flow, but because of the pendulum volume, the effect of pump removal on PVA is equivalent to the PVA at $1.8 \mathrm{~L} / \mathrm{min}$ positive mean flow.

pattern can be seen in the PV loop. In comparison with rotary pumps, every second beat would be massively unloaded (corresponding to full unloading with a rotary pump) and the intervening beat would be far less loaded than without a pump because of the reduced mean atrial pressure.

\section{Discussion}

During the last 4 years, continuous flow pumps have proven their ability to provide full restoration of organ function in patients with terminal heart failure and to sustain support for extended time periods. ${ }^{9,11,18-21}$ These systems are already a real alternative to pulsatile devices, offering a number of advantages concerning size, blood-contacting surface, simplicity, lack of venting tubes (and therefore the ability to use more flexible cables), and far fewer infections in the experience of our center. (As of April 2003, 15 patients have left the hospital while supported, 14 have undergone transplantation, 2 currently remain on support in the hospital, and 10 have died.) 


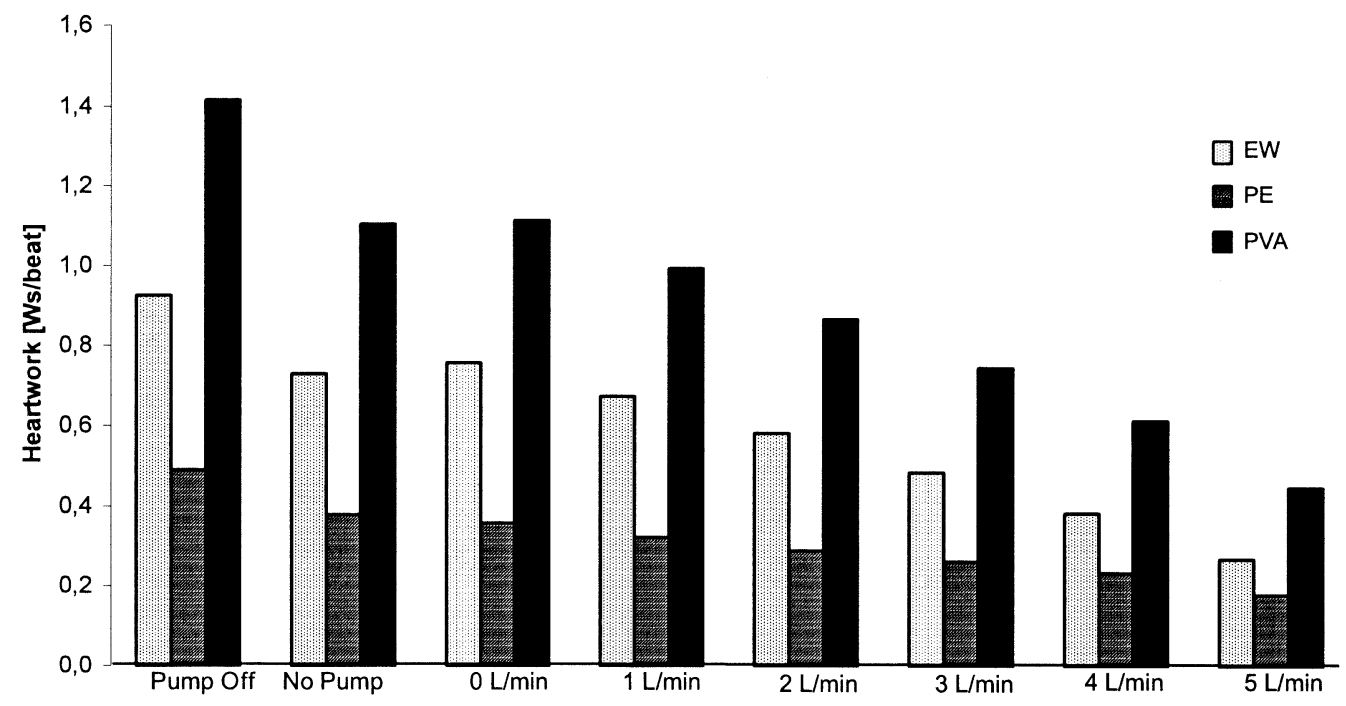

Figure 6. EW, PE, and PVA for paracorporeally placed rotary pump with long inflow and outflow cannulas (both $1 \mathrm{~m})$. Because of the lower pendulum volume resulting from the damping effect of the tubing, the PVA without pump is equivalent to the situation at zero flow through the paracorporeal pump.
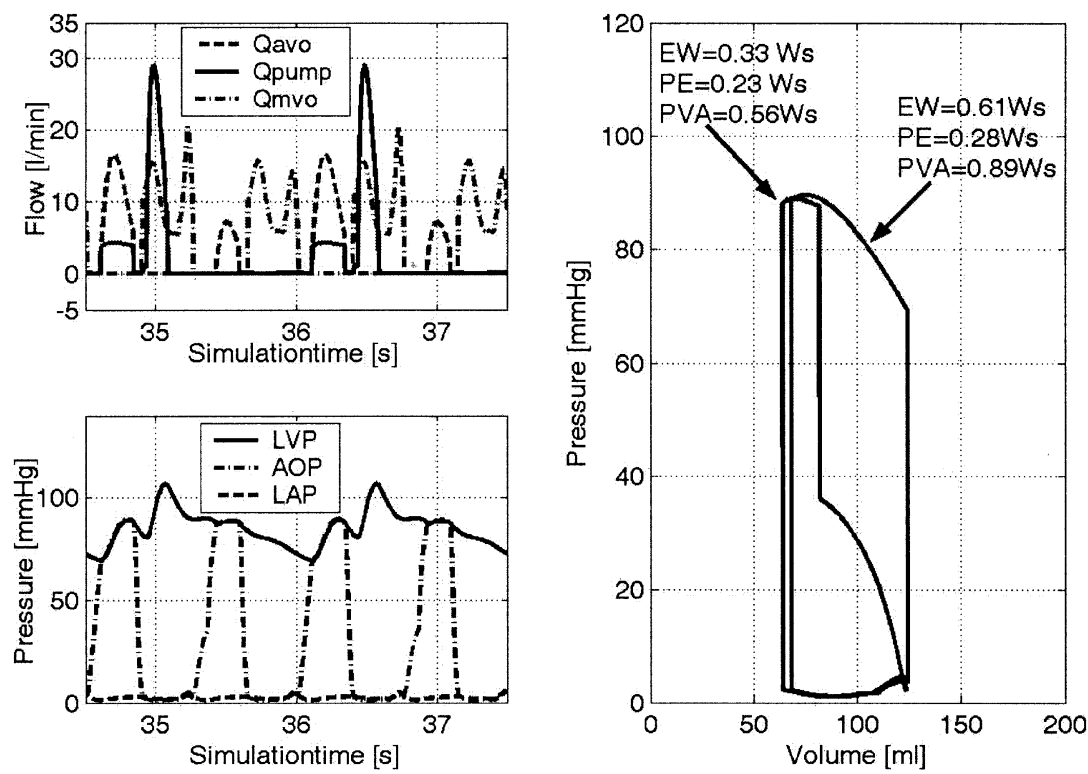

Figure 7. Pressure and flow patterns and PV loops for a pulsatile assist device in 1:1 assist mode (resulting mean pump flow $2.55 \mathrm{~L} / \mathrm{min}$ ). The pump supports every second heart beat, leading to a complex shape of the PV loop.

Non-occlusiveness has been discussed intensively because of the safety threat it poses in case of pump stoppage. ${ }^{22,23}$ Certainly, backflow imposes an increased volume load on the left ventricle, typically in the range of 1 to 2 $\mathrm{L} / \mathrm{min}$ (mean value) with an implanted pump. This load is similar to that imposed by a moderate grade of insufficiency in the aortic valve. ${ }^{24}$ On the other hand, backflow offers the advantage of washout and avoidance of thrombus growth, effects that increase the time for reaction to pump stoppage if the heart has sufficiently recovered to withstand the unassisted period. ${ }^{11}$

In addition, an unobstructed flow path provides new approaches to weaning in case of cardiac recovery, allowing reduction of support to very low or even negative values. This approach is impossible with pulsatile pumps, in which flow less than 2.5 to $2 \mathrm{~L} / \mathrm{min}$ would lead to thrombus formation. Sophisticated protocols have been developed for weaning patients from pulsatile devices. ${ }^{1-4}$ The procedure 
usually involves several steps of flow reduction, using increasing anticoagulation, with initial stoppage occurring during several minutes under catheterization, echo control, or both. However, because the heart cannot be trained at minimal support values for extended time periods, the decision to explant these devices is very difficult because the process immediately subjects the heart to a considerable additional load. ${ }^{25}$

With rotary pumps, flow can be reduced to minimal values, imposing only the risk of multiple reiterating passages of pendular flow through the pump, which may increase hemolysis. However, as demonstrated in this article, a mean flow of zero is not equivalent to a removed or occluded pump. Although this pendular blood volume does not create any hydraulic energy in the arterial system (except viscous losses), it does create additional area in the PV loop and therefore increases metabolic demand.

For weaning purposes, it would be interesting to know the pump speed and corresponding level of flow support that would be equivalent to the situation after pump removal. The simulation presented in this article predicts that a support level of approximately $30 \%$ of the whole cardiac output, with the pump in place, would be equivalent to the workload after explant. Mean rotary pump flows less than $1.5 \mathrm{~L} / \mathrm{min}$ (in an average adult equivalent to $<25 \%$ of normal cardiac output) would train a patient's heart to a situation exceeding that imposed by pump removal.

This numeric result clearly requires testing in vivo even though it is based on the well-accepted theory of PV loops, which states the PVA to be equivalent to metabolic demand. ${ }^{14,15}$ The unusual situation of continuous unloading, paradoxic effects to the right ventricle because of septal movements, and inaccuracies in the definition of PVA in the case of decompensating ventricles with nonlinear end-systolic pressure relationships ${ }^{13}$ may all influence the actual result. Notwithstanding these concerns, this study clearly predicts that implanted rotary pumps with short cannulas provide a far better transition to full ventricular load than either paracorporeally placed pumps with long cannulas or pulsatile pumps. ${ }^{26}$ The latter require minimal flow to prevent thromboembolism and provide variable unloading from beat to beat. Rotary pumps also permit anticipating, for extended time periods, the increased load the heart will experience after explant. This feature may be important in choosing pumps intended for temporary assist in combination with other adjuvant therapies. This study does not account for changing conditions in the right ventricle, which is certainly influenced, at least, by the changing load on the intraventricular septum.

On the other hand, this study suggests that pulsatile pumps have a greater potential for ventricular unloading from the metabolic viewpoint. Pulsatile assist might be preferred when partial assistance is needed in the early postoperative period. However, in this situation the pump usually carries the full flow and the aortic valve stays closed. Nonetheless, pulsatility could be desirable when the aortic valve opens after initial recovery.

Of course, this computer study also contains some inherent limitations: The heart was modeled without additional comorbidities, especially disturbances in diastolic relaxation or valve insufficency, which may well occur in recovering hearts. Such deficiencies may influence the quantitative results but should not alter the general trends. Further, it has not yet been proven that axial pumps can be run at low flows without increase of thromboembolic risk, but the experiences with extended switch-off of pumps and the recent application of a MicroMed DeBakey ventricular assist device in a child cause optimism for a proper performance of these pumps also at low-flow rates.

\section{Conclusion}

Unloading of the native ventricle by implanted rotary pumps is influenced by an oscillating flow caused by interaction between the pump and ventricle. This flow imposes an additional metabolic demand that can be evaluated from changes in the area under a PV curve. The additional load cancels the benefit of pump support to the heart at ratios of pump flow to systemic flow of $25 \%$ to $30 \%$. This effect suggests effective means of weaning the heart from support before pump removal.

We thank Professor Edward Leonard of Columbia University, currently a Fulbright Guest Professor at our institution, for contributions to the discussion of this work.

\section{References}

1. Müller J, Wallukat G, Weng Y, Dandel M, Ellinghaus P, Huetter J, et al. Predictive factors for weaning from a cardiac assist device. An analysis of clinical, gene expression, and protein data. J Heart Lung Transplant. 2001;20:202.

2. Slaughter MS, Silver MA, Farrar DJ, Tatooles AJ, Pappas PS. A new method of monitoring recovery and weaning the Thoratec left ventricular assist device. Ann Thorac Surg. 2001;71:215-8.

3. Milting H, EL Banayosy A, Kassner A, Fey O, Sarnowski P, Arusoglu $\mathrm{L}$, et al. The time course of natriuretic hormones as plasma markers of myocardial recovery in heart transplant candidates during ventricular assist device support reveals differences among device types. J Heart Lung Transplant. 2001;20:949-55.

4. Acker MA. Mechanical circulatory support for patients with acutefulminant myocarditis. Ann Thorac Surg. 2001;71(3 Suppl):S73-6; discussion S82-5. Review.

5. Anderson DW. Blood pumps: technologies and markets in transformation. Artif Organs. 2001;25:406-10.

6. Kocher AA, Schuster MD, Szabolcs MJ, Takuma S, Burkhoff D, Wang J, et al. Neovascularization of ischemic myocardium by human bone-marrow-derived angioblasts prevents cardiomyocyte apoptosis, reduces remodeling and improves cardiac function. Nat Med. 2001;7: 430-6.

7. Eizema K, Fechner H, Bezstarosti K, Schneider-Rasp S, van der Laarse A, Wang H, et al. Adenovirus-based phospholamban antisense expression as a novel approach to improve cardiac contractile dysfunction: comparison of a constitutive viral versus an endothelin-1responsive cardiac promoter. Circulation. 2000;101:2193-9. 
8. Termuhlen DF, Swartz MT, Ruzevich SA, Reedy JE, Pennington DG. Hemodynamic predictors for weaning patients from ventricular assist devices (VADs). J Biomater Appl. 1990;4:374-90.

9. Wieselthaler GM, Schima H, Hiesmayr M, Pacher R, Laufer G, Noon GP, et al. First clinical experience with the DeBakey VAD continuousaxial-flow pump for bridge to transplantation. Circulation. 2000;101: 356-9.

10. Potapov EV, Loebe M, Nasseri BA, Sinawski H, Koster A, Kuppe H, et al. Pulsatile flow in patients with a novel nonpulsatile implantable ventricular assist device. Circulation. 2000;102(19 Suppl 3):III183-7.

11. Frazier OH, Gregoric ID, Delgado RM, Myers TJ, Radovancevic B, Khan T, et al. Initial experience with the Jarvik 2000 left ventricular assist system as a bridge to transplantation: report of 4 cases. $J$ Heart Lung Transplant. 2001;20:201.

12. Westaby S, Banning AP, Jarvik R, Frazier OH, Pigott DW, Jin XY, et al. First permanent implant of the Jarvik 2000 Heart. Lancet. 2000; 356:900-3

13. Vollkron M, Schima H, Huber L, Wieselthaler G. Interaction of the cardiovascular system with an implanted rotary blood pump: simulation study with a refined computer model. Artif Organs. 2002;26:4.

14. Suga H. Cardiac mechanics and energetics-from Emax to PVA. Front Med Biol Eng. 1990;2:3-22.

15. Araki J, Shimizu J, Mikane T, Mohri S, Matsubara H, Yamaguchi H, et al. Ventricular pressure-volume area (PVA) accounts for cardiac energy consumption of work production and absorption. Adv Exp Med Biol. 1998;453:491-8 Review.

16. Donovan FM Jr. Design of a hydraulic analog of the circulatory system for evaluating artificial hearts. Biomater Med Devices Artif Organs. 1975;3:439-49.

17. Sagawa K, Maughan L, Suga H, Sunagawa K. Cardiac contraction and the pressure-volume relationship. Oxford: Oxford University Press; 1988.
18. Wieselthaler GM, Schima H, Dworschak M, Quittan M, Nuhr M, Czerny M, et al. First experiences with outpatient care of patients with implanted axial flow pumps. Artif Organs. 2001;25:331-5.

19. Wilhelm MJ, Hammel D, Schmid C, Stypmann J, Asfour B, Kemper $\mathrm{D}$, et al. Clinical experience with nine patients supported by the continuous flow Debakey VAD. J Heart Lung Transplant. 2001;20: 201.

20. Noon GP, Morley DL, Irwin S, Abdelsayed SV, Benkowski RJ, Lynch BE. Clinical experience with the MicroMed DeBakey ventricular assist device. Ann Thorac Surg. 2001;71(3 Suppl):S133-8.

21. Vitali E, Lanfranconi M, Ribera E, Bruschi G, Colombo T, Frigerio M, et al. Successful experience in bridging patients to heart transplantation with the MicroMed DeBakey ventricular assist device. Ann Thorac Surg. 2003;75:1200-4.

22. Ohtsuka G, Yoshikawa M, Sueoka A, Saito K, Igarashi A, Tayama E, et al. An emergency balloon occlusion system for a rotary blood pump left ventricular assist system. Artif Organs. 1999;23:704-7.

23. Nakata KI, Shiono M, Akiyama K, Orime Y, Saito A, Tutomu H, et al. The estimation of cardiac function from the rotary blood pump. Artif Organs. 2001;25:709-12.

24. Vandenberghe S, Segers P, Meyns B, Verdonck PR. Effect of rotary blood pump failure on left ventricular energetics assessed by mathematical modeling. Artif Organs. 2002;26:1032-9.

25. Helman DN, Maybaum SW, Morales DL, Williams MR, Beniaminovitz A, Edwards NM, et al. Recurrent remodeling after ventricular assistance: is long-term myocardial recovery attainable? Ann Thorac Surg. 2000;70:1255-8.

26. Noon GP, Lafuente JA, Irwin S. Acute and temporary ventricular support with BioMedicus centrifugal pump. Ann Thorac Surg. 1999; 68:650-4. 